\title{
Relaxation time for the alignment between the spin of a finite-mass quark or antiquark and the thermal vorticity in relativistic heavy-ion collisions
}

\author{
Alejandro Ayala $\odot,{ }^{1,2}$ David de la Cruz $\odot{ }^{3}$ L. A. Hernández $\odot,{ }^{1,2,4}$ and Jordi Salinas $\oplus^{1 *}$ \\ ${ }^{1}$ Instituto de Ciencias Nucleares, Universidad Nacional Autónoma de México, \\ Apartado Postal 70-543, México Distrito Federal 04510, Mexico \\ ${ }^{2}$ Centre for Theoretical and Mathematical Physics, and Department of Physics, \\ University of Cape Town, Rondebosch 7700, South Africa \\ ${ }^{3}$ Departamento de Física, Escuela Superior de Física y Matemáticas del Instituto Politécnico Nacional, \\ Unidad Adolfo López Mateos, Edificio 9, 07738 Ciudad de México, México \\ ${ }^{4}$ Facultad de Ciencias de la Educación, Universidad Autónoma de Tlaxcala, Tlaxcala 90000, Mexico
}

(Received 19 May 2020; accepted 1 September 2020; published 17 September 2020)

\begin{abstract}
We study the relaxation time required for the alignment between the spin of a finite-mass quark or antiquark and the thermal vorticity, at finite temperature and baryon chemical potential, in the context of relativistic heavy-ion collisions. The relaxation time is computed as the inverse of the total reaction rate that in turn is obtained from the imaginary part of the quark or antiquark self-energy. We model the interaction between spin and thermal vorticity within the medium by means of a vertex coupling quarks and thermal gluons that, for a uniform temperature, is proportional to the global angular velocity and inversely proportional to the temperature. We use realistic estimates for the angular velocities for different collision energies and show that the effect of the quark mass is to reduce the relaxation times as compared to the massless quark case. Using these relaxation times we estimate the intrinsic quark and antiquark polarizations produced by the thermal vorticity. We conclude by pointing out that, although the intrinsic global polarization of the $s$-quark turns out to be larger than that of the $\bar{s}$-quark, there is room to explain recent STAR results which show that the global polarization of $\bar{\Lambda}$ is larger than that of $\Lambda$ when considering that these hyperons can be produced from different density regions in a core-corona model.
\end{abstract}

DOI: $10.1103 /$ PhysRevD.102.056019

\section{INTRODUCTION}

Results from heavy-ion collisions experiments have contributed significantly to our understanding of the properties of strongly interacting matter at high temperature and density. In these reactions, two atomic nuclei collide at relativistic energies producing a deconfined state of hadronic matter, the so-called quark-gluon plasma (QGP). Although many properties of this state have been revealed by means of a number of different probes, it is also fair to say that others still remain elusive at large. One of these has to do with the possibility to create a vortical fluid in peripheral collisions. Were this to be the case, the most promising way to elucidate its properties is by means of the alignment of particle spin to the global angular momentum, which in turn could be detected measuring a nonvanishing global particle

jordissm@ciencias.unam.mx

Published by the American Physical Society under the terms of the Creative Commons Attribution 4.0 International license. Further distribution of this work must maintain attribution to the author(s) and the published article's title, journal citation, and DOI. Funded by SCOAP ${ }^{3}$. polarization. This possibility has prompted the search for global polarization of hadrons, most notably of $\Lambda$ and $\bar{\Lambda}$ [1-16]. The STAR Beam Energy Scan (BES) program has measured the $\Lambda$ and $\bar{\Lambda}$ global polarizations as functions of the collision energy [17-19] showing that as the latter decreases, the $\bar{\Lambda}$ polarization rises more steeply than the $\Lambda$ polarization. This intriguing result motivates the search for a deeper understanding of the conditions for the relaxation between angular momentum and spin degrees of freedom and of its dependence on the collision parameters such as energy, impact parameter, temperature, and baryon chemical potential.

In a previous work [20], we studied the relaxation time for spin and thermal vorticity alignment in a QGP at finite temperature $T$ and quark chemical potential $\mu_{q}=\mu_{B} / 3$, where $\mu_{B}$ is the baryon chemical potential. For these purposes, we resorted to the computation of the quark self-energy where the interaction with thermal gluons is mediated by a phenomenological vertex that couples the thermal vorticity to spin. To make matters simpler, we performed the calculation for massless quarks whose momentum was small compared to $T$ and/or $\mu_{q}$. In this work we remove such approximations and compute the 
relaxation time for massive quarks with arbitrary momentum. We show that the effect of accounting for the quark mass produces that the interaction rate is larger which in turn translates into a smaller relaxation time, as compared to the massless quark case. Other attempts to compute the relaxation time using different approaches have been reported in Refs. [21-23].

This work is organized as follows. In Sec. II we calculate the interaction rate for a massive quark at finite density and temperature. In Sec. III we show the results obtained for the quark and antiquark relaxation times as functions of temperature, collision energy, and quark intrinsic global polarization. Finally, Sec. IV provides a summary along with a discussion on the consequences of this calculation for hyperon polarization.

\section{QUARK INTERACTION RATE AT FINITE DENSITY AND TEMPERATURE}

Consider a QCD plasma in thermal equilibrium at temperature $T$ and quark chemical potential $\mu_{q}$. The interaction rate $\Gamma$ of a quark with four-momentum $P=\left(p_{0}, \vec{p}\right)$ can be expressed in terms of the quark selfenergy $\Sigma$ as

$$
\Gamma\left(p_{0}\right)=\tilde{f}\left(p_{0}-\mu_{q}\right) \operatorname{Tr}\left\{\gamma^{0} \operatorname{Im} \Sigma\right\}
$$

with $\tilde{f}\left(p_{0}-\mu_{q}\right)$ being the Fermi-Dirac distribution. The interaction between the thermal vorticity and the quark spin is modeled by means of an effective vertex

$$
\lambda_{a}^{\mu}=g \frac{\sigma^{\alpha \beta}}{2} \bar{\omega}_{\alpha \beta} \gamma^{\mu} t_{a}
$$

where $\sigma^{\alpha \beta}=\frac{i}{2}\left[\gamma^{\alpha}, \gamma^{\beta}\right]$ is the quark spin operator and $t_{a}$ are the color matrices in the fundamental representation. This vertex builds on the ideas discussed in Ref. [24] and the subsequent studies of Refs. [6,25-28] and references therein. In order to present a self-contained discussion, we hereby spell out the ingredients needed for the computation, highlighting the new elements as compared to Ref. [20]. Recall that the thermal vorticity is defined as [29]

$$
\bar{\omega}_{\mu \nu}=\frac{1}{2}\left(\partial_{\nu} \beta_{\mu}-\partial_{\mu} \beta_{\nu}\right),
$$

where $\beta_{\mu}=u_{\mu}(x) / T(x)$, with $u_{\mu}(x)$ the local fluid fourvelocity and $T(x)$ the local temperature. Thermal vorticity is produced in peripheral collisions where the colliding matter develops a global angular velocity $\vec{\omega}=\omega \hat{z}$, normal to the reaction plane, that for our purposes is chosen as the direction of the $\hat{z}$ axis. The orbital angular momentum is due to the inhomogeneity of the matter density profile in the transverse plane [28]. For a constant angular velocity and uniform temperature, the magnitude of the thermal vorticity is given by $\omega / T$.

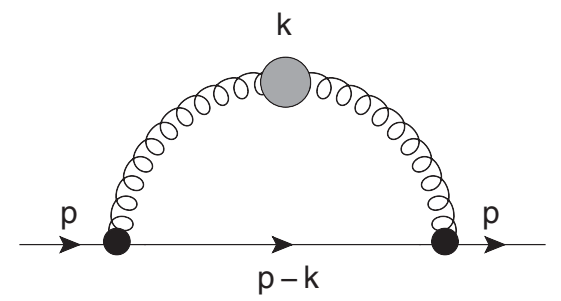

FIG. 1. One-loop quark self-energy diagram that defines the kinematics. The gluon line with a blob represents the effective gluon propagator at finite density and temperature. The blobs on the quark-gluon vertices represent the effective coupling between the quark spin and the vorticity.

The one-loop contribution to $\Sigma$, depicted in Fig. 1, is given explicitly by

$$
\Sigma=T \sum_{n} \int \frac{d^{3} k}{(2 \pi)^{3}} \lambda_{a}^{\mu} S(\not P-\not K) \lambda_{b}^{\nu *} G_{\mu \nu}^{a b}(K)
$$

where $S$ and ${ }^{*} G$ are the quark and effective gluon propagators, respectively. The effective gluon propagator is obtained by summing the geometric series for the oneloop gluon polarization tensor at high temperature and/or quark chemical potential. The intermediate quark line is taken as a bare quark propagator such that the inverse of the interaction rate corresponds to the relaxation time for the spin and vorticity alignment for quarks that are originally not thermalized.

In a covariant gauge, the hard thermal loop (HTL) approximation to the effective gluon propagator is given by

$$
{ }^{*} G_{\mu \nu}(K)={ }^{*} \Delta_{L}(K) P_{L \mu \nu}+{ }^{*} \Delta_{T}(K) P_{T \mu \nu}
$$

where $P_{L, T \mu \nu}$ are the polarization tensors for threedimensional longitudinal and transverse gluons, both of which are, of course, four-dimensionally transverse. The gluon propagator functions for longitudinal and transverse modes, ${ }^{*} \Delta_{L, T}(K)$, are given by

$$
\begin{aligned}
{ }^{*} \Delta_{L}(K)^{-1}= & K^{2}+2 m^{2} \frac{K^{2}}{k^{2}}\left[1-\left(\frac{i \omega_{n}}{k}\right) Q_{0}\left(\frac{i \omega_{n}}{k}\right)\right], \\
{ }^{*} \Delta_{T}(K)^{-1}= & -K^{2}-m^{2}\left(\frac{i \omega_{n}}{k}\right)\left\{\left[1-\left(\frac{i \omega_{n}}{k}\right)^{2}\right]\right. \\
& \left.\times Q_{0}\left(\frac{i \omega_{n}}{k}\right)+\left(\frac{i \omega_{n}}{k}\right)\right\},
\end{aligned}
$$

where

$$
Q_{0}(x)=\frac{1}{2} \ln \frac{x+1}{x-1}
$$

and $m$ is the gluon thermal mass given by 


$$
m^{2}=\frac{1}{6} g^{2} C_{A} T^{2}+\frac{1}{12} g^{2} C_{F}\left(T^{2}+\frac{3}{\pi^{2}} \mu^{2}\right),
$$

where $C_{A}=3$ and $C_{F}=4 / 3$ are the Casimir factors for the adjoint and fundamental representations of $S U(3)$, respectively.

The sum over Matsubara frequencies involves products of the propagator functions for longitudinal and transverse gluons ${ }^{*} \Delta_{L, T}$ and the Matsubara propagator for the bare quark $\tilde{\Delta}_{F}$, such that the term that depends on the summation index can be expressed as

$$
S_{L, T}=T \sum_{n}{ }^{*} \Delta_{L, T}\left(i \omega_{n}\right) \tilde{\Delta}_{F}\left(i\left(\omega_{m}-\omega_{n}\right)\right) .
$$

This sum is more straightforwardly evaluated introducing the spectral densities $\rho_{L, T}$ and $\tilde{\rho}$ for the gluon and fermion propagators, respectively. The imaginary part of $S_{i}(i=L, T)$ can thus be written as

$$
\begin{aligned}
\operatorname{Im} S_{i}= & \pi\left(e^{\left(p_{0}-\mu_{q}\right) / T}+1\right) \int_{-\infty}^{\infty} \frac{d k_{0}}{2 \pi} \int_{-\infty}^{\infty} \frac{d p_{0}^{\prime}}{2 \pi} f\left(k_{0}\right) \\
& \times \tilde{f}\left(p_{0}^{\prime}-\mu\right) \delta\left(p_{0}-k_{0}-p_{0}^{\prime}\right) \rho_{i}\left(k_{0}\right) \tilde{\rho}\left(p_{0}^{\prime}\right),
\end{aligned}
$$

where $f\left(k_{0}\right)$ is the Bose-Einstein distribution. The spectral densities $\rho_{L, T}\left(k_{0}, k\right)$ are obtained from the imaginary part of ${ }^{*} \Delta_{L, T}\left(i \omega_{n}, k\right)$ after the analytic continuation $i \omega_{n} \rightarrow$ $k_{0}+i \epsilon$ and contain the discontinuities of the gluon propagator across the real $k_{0}$ axis. Their support depends on the ratio $x=k_{0} / k$. For $|x|>1, \rho_{L, T}$ have support on the (timelike) quasiparticle poles. For $|x|<1$ their support coincides with the branch cut of $Q_{0}(x)$. On the other hand, the spectral density corresponding to a bare quark is given by

$$
\tilde{\rho}\left(p_{0}^{\prime}\right)=2 \pi \epsilon\left(p_{0}^{\prime}\right) \delta\left(p_{0}^{\prime 2}-E_{p}^{2}\right)
$$

where $E_{p}^{2}=(p-k)^{2}+m_{q}^{2}$ with $m_{q}$ the quark mass. The kinematical restriction that Eq. (11) imposes on Eq. (10) limits the integration over gluon energies to the spacelike region, namely, $|x|<1$. Therefore, the parts of the gluon spectral densities that contribute to the interaction rate are given by

$$
\begin{aligned}
& \rho_{L}\left(k_{0}, k\right)=\frac{x}{1-x^{2}} \frac{2 \pi m^{2} \theta\left(k^{2}-k_{0}^{2}\right)}{\left[k^{2}+2 m^{2}\left(1-\frac{x}{2} \ln \left|\frac{1+x}{1-x}\right|\right)\right]^{2}+\left[\pi m^{2} x\right]^{2}}, \\
& \rho_{T}\left(k_{0}, k\right)=\frac{\pi m^{2} x\left(1-x^{2}\right) \theta\left(k^{2}-k_{0}^{2}\right)}{\left[k^{2}\left(1-x^{2}\right)+m^{2}\left(x^{2}+(x / 2)\left(1-x^{2}\right) \ln \left|\frac{1+x}{1-x}\right|\right)\right]^{2}+\left[(\pi / 2) m^{2} x\left(1-x^{2}\right)\right]^{2}} .
\end{aligned}
$$

Collecting all the ingredients, the interaction rate for a massive quark with energy $p_{0}$ to align its spin with the thermal vorticity is given by

$$
\begin{aligned}
\Gamma\left(p_{0}\right)= & \frac{\alpha_{s}}{4 \pi}\left(\frac{\omega}{T}\right)^{2} \frac{C_{F}}{\sqrt{p_{0}^{2}-m_{q}^{2}}} \int_{0}^{\infty} d k k \int_{\mathcal{R}} d k_{0}\left[1+f\left(k_{0}\right)\right] \\
& \times \tilde{f}\left(p_{0}+k_{0}-\mu_{q}\right) \sum_{i=L, T} C_{i}\left(p_{0}, k_{0}, k\right) \rho_{i}\left(k_{0}, k\right),
\end{aligned}
$$

where $\mathcal{R}$ represents the region

$$
\begin{aligned}
& k_{0} \geq \sqrt{\left(\sqrt{p_{0}^{2}-m_{q}^{2}}-k\right)^{2}+m_{q}^{2}}-p_{0}, \\
& k_{0} \leq \sqrt{\left(\sqrt{p_{0}^{2}-m_{q}^{2}}+k\right)^{2}+m_{q}^{2}}-p_{0} .
\end{aligned}
$$

It can be checked that the region of integration over $k_{0}$ in Eq. (14) reduces to $-k \leq k_{0} \leq k$ when $m_{q} \rightarrow 0$, as was obtained in Ref. [20]. Notice that Eq. (14) implies that the available phase space is reduced in the massive quark case, as one could expect. The polarization coefficients $C_{L, T}$ come from the contraction of the polarization tensors $P_{L, T \mu \nu}$ with the trace of the factors involving Dirac gamma matrices from the self-energy. After implementing the kinematical restrictions for the allowed values of the angle between the quark and gluon momenta, these functions are found to be

$$
\begin{aligned}
C_{T}\left(p_{0}, k_{0}, k\right)= & 8\left(p_{0}+k_{0}\right) \mathcal{C}\left(p_{0}, k_{0}, k\right) \\
C_{L}\left(p_{0}, k_{0}, k\right)= & -8\left(p_{0}+k_{0}\right)\left[\mathcal{C}\left(p_{0}, k_{0}, k\right)-\frac{1}{2}\right] \\
& -8 \frac{p_{0} k^{2}}{k_{0}^{2}-k^{2}} \mathcal{C}\left(p_{0}, k_{0}, k\right)
\end{aligned}
$$

with

$$
\mathcal{C}\left(p_{0}, k_{0}, k\right)=\left(\frac{k^{2}-2 k_{0} p_{0}-k_{0}^{2}}{2 k \sqrt{p_{0}^{2}-m_{q}^{2}}}\right)^{2}
$$


This result should be contrasted with Eq. (14) of Ref. [20], which was computed for the massless and small quark momentum limit. The total interaction rate is obtained by integrating Eq. (13) over the available phase space

$$
\Gamma=V \int \frac{d^{3} p}{(2 \pi)^{3}} \Gamma\left(p_{0}\right)
$$

where $V$ is the volume of the overlap region in the collision. Recall that, for the collision of symmetric systems of nuclei with radii $R$ and a given impact parameter $b, V$ is given by

$$
V=\frac{\pi}{3}(4 R+b)(R-b / 2)^{2} .
$$

Following the method outlined in Ref. [30], the initial angular velocity $\omega$ (i.e., after full nuclei overlap) produced in $\mathrm{Au}+\mathrm{Au}$ collisions is hereby computed as follows: For every event generated with UrQMD [31], a smearing function is used to smooth the position profile of the participating nucleons, which then can be used to obtain a continuous velocity field. With this information, the angular velocity can be computed as the usual spatial derivative of the velocity field. Finally, only the angular velocity at the spatial origin is used. This procedure is repeated for $10^{4}$ events for every collision energy and impact parameter. The results are then averaged. The calculation inputs are the nucleons position and momenta. With this procedure, the computed values of $\omega$ correspond to initial angular velocities. The volume given by Eq. (18) serves as an approximation to the geometry of the interaction region, but takes no part on the process to obtain $\omega$ other than to represent an analytical proxy to describe the overlap region during the collision.

The results of our simulations for the initial angular velocity are shown in Fig. 2 for three different impact

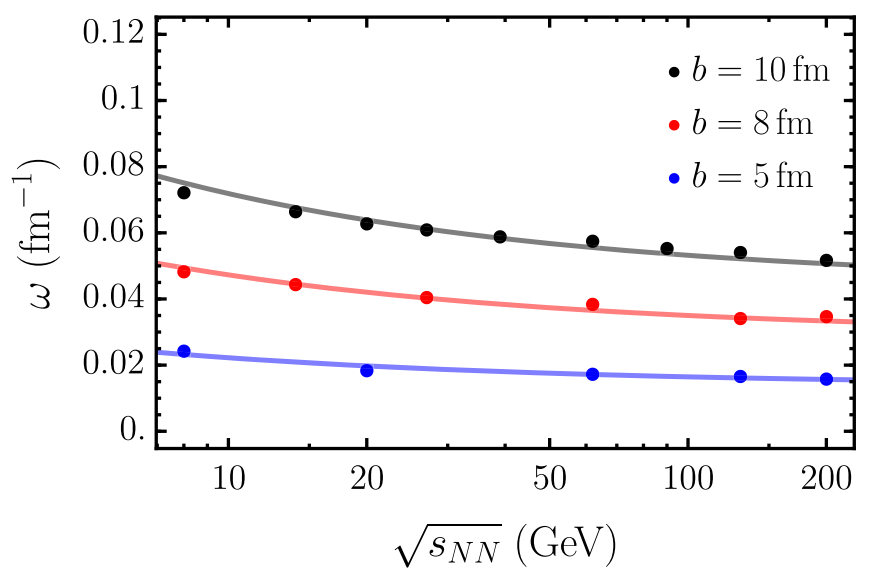

FIG. 2. Initial angular velocity $\omega$ for $\mathrm{Au}+\mathrm{Au}$ collisions at impact parameters $b=5,8,10 \mathrm{fm}$ as functions of collision energy $\sqrt{s_{N N}}$. Solid lines are the fit of the UrQMD results, using Eq. (19). parameters, $b=5,8,10 \mathrm{fm}$. Solid lines are the fit of the data using the functional form

$$
\omega=\frac{\omega_{0}}{2} \frac{b^{2}}{V_{N}}\left[1+2\left(\frac{m_{N}}{\sqrt{s_{N N}}}\right)^{1 / 2}\right],
$$

where $V_{N}=(4 \pi / 3) R^{3}, R=1.1 A^{1 / 3}$, and $\omega_{0}$ is the free parameter of the fit; in all cases $\omega_{0} \simeq 1$. Notice that, as explored in Ref. [30], different choices of smearing functions for the calculation of the velocity profile, or the definition of the velocity of the produced particles itself, can lead to a variation on the values resulting for the angular velocity; for a more detailed study, see Refs. [32,33]. From the expression for $\Gamma$ in Eq. (17), we study the parametric dependence of the relaxation time for spin and vorticity alignment, defined as

$$
\tau \equiv 1 / \Gamma
$$

We now proceed to present the results for the quark and antiquark relaxation times as computed with Eq. (20) as functions of temperature and collision energy as well as the intrinsic quark global polarization as a function of time.

\section{RESULTS AND DISCUSSION}

To explicitly compute the relaxation times, we hereby use the value for the strong coupling $\alpha_{s}=0.3$. Figure 3 shows the relaxation time $\tau$ for massive quarks with $m_{q}=100 \mathrm{MeV}$, corresponding to the strange quark mass, contrasted with the massless quarks case, as a function of temperature $T$, for semicentral collisions at an impact parameter $b=10 \mathrm{fm}$ for two different values of quark chemical potential $\mu_{q}$ and collision energy $\sqrt{s_{N N}}$. In both cases, $\omega$ is computed using our simulations described

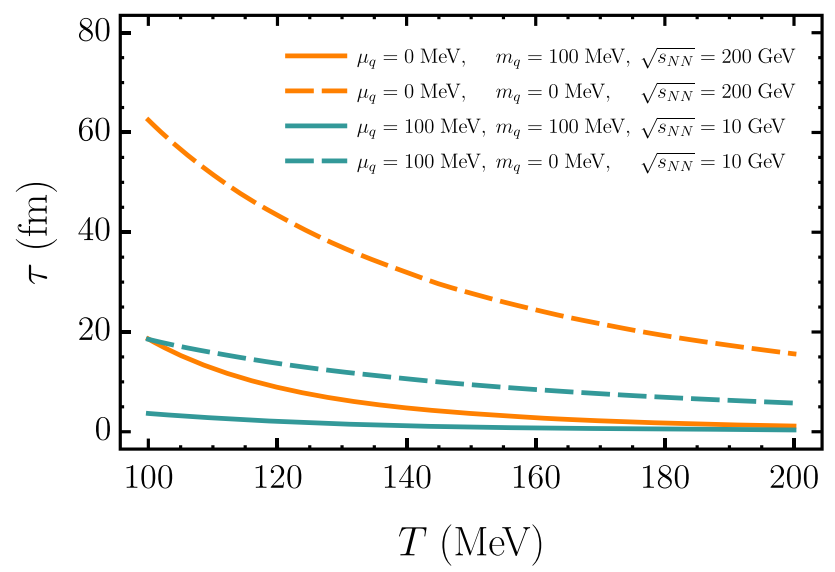

FIG. 3. Relaxation time $\tau$ for quarks as a function of temperature $T$ for semicentral collisions at an impact parameter $b=$ $10 \mathrm{fm}$ for $\sqrt{s_{N N}}=10,200 \mathrm{GeV}$ with $\omega \simeq 0.072,0.051 \mathrm{fm}^{-1}$, respectively. In dashed lines massless quarks [20], in solid lines massive quarks. 


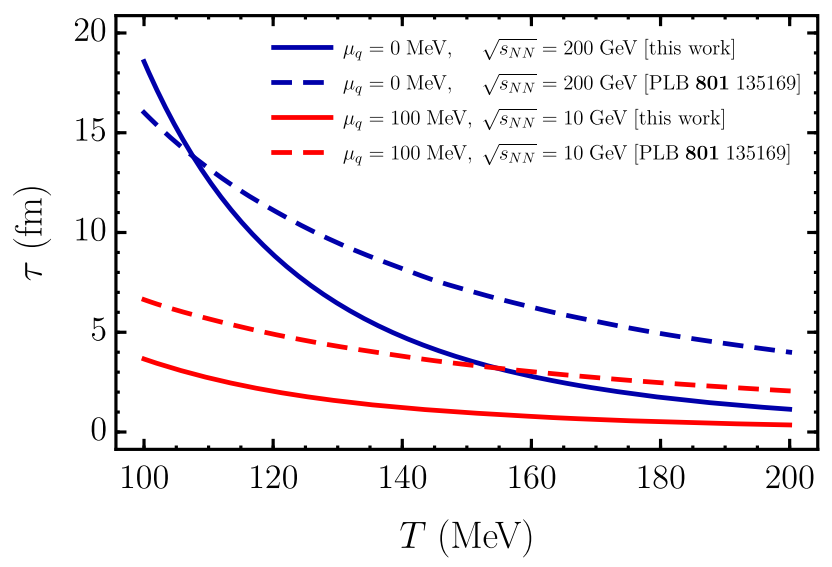

FIG. 4. Relaxation time $\tau$ for quarks as a function of temperature $T$ for semicentral collisions at an impact parameter $b=$ $10 \mathrm{fm}$ for $\sqrt{s_{N N}}=10,200 \mathrm{GeV}$ with $\omega \simeq 0.12,0.10 \mathrm{fm}^{-1}$, respectively. In dashed lines massless quarks [20], in solid lines massive quarks.

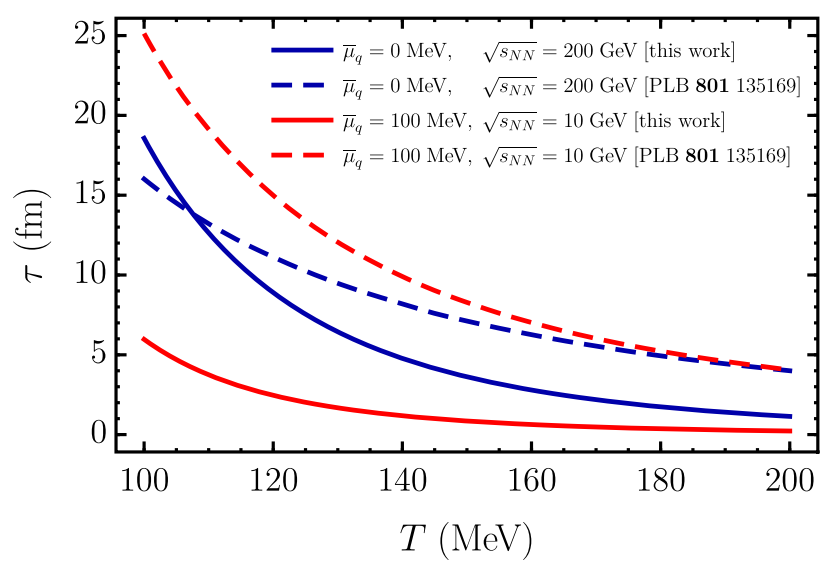

FIG. 5. Relaxation time $\bar{\tau}$ for antiquarks as a function of temperature $T$ for semicentral collisions at an impact parameter $b=10 \mathrm{fm}$ for $\sqrt{s_{N N}}=10,200 \mathrm{GeV}$ with $\omega \simeq 0.12,0.10 \mathrm{fm}^{-1}$, respectively. In dashed lines massless quarks [20], in solid lines massive quarks.

above. The effect of quark mass and of relaxing the assumption of a small quark energy is to reduce the relaxation time for the entire range of considered temperatures, as compared to the massless case. This may seem counterintuitive given that a finite quark mass reduces the available phase space. However, notice that the new terms in Eq. (15), as compared to Eq. (14) of Ref. [20], compensate this reduction and contribute significantly to a higher interaction rate. Figure 4 shows the temperature dependence of the relaxation time for quarks, for two different collision energies and quark chemical potentials at an impact parameter $b=10 \mathrm{fm}$. For direct comparison to Ref. [20], the values of $\omega \simeq 0.12,0.10 \mathrm{fm}^{-1}$ are used for the massless case. Notice that $\tau \lesssim 5 \mathrm{fm}$ for the temperature range $150 \mathrm{MeV}<T<200 \mathrm{MeV}$, where the phase transition is expected to occur. In this temperature range, the

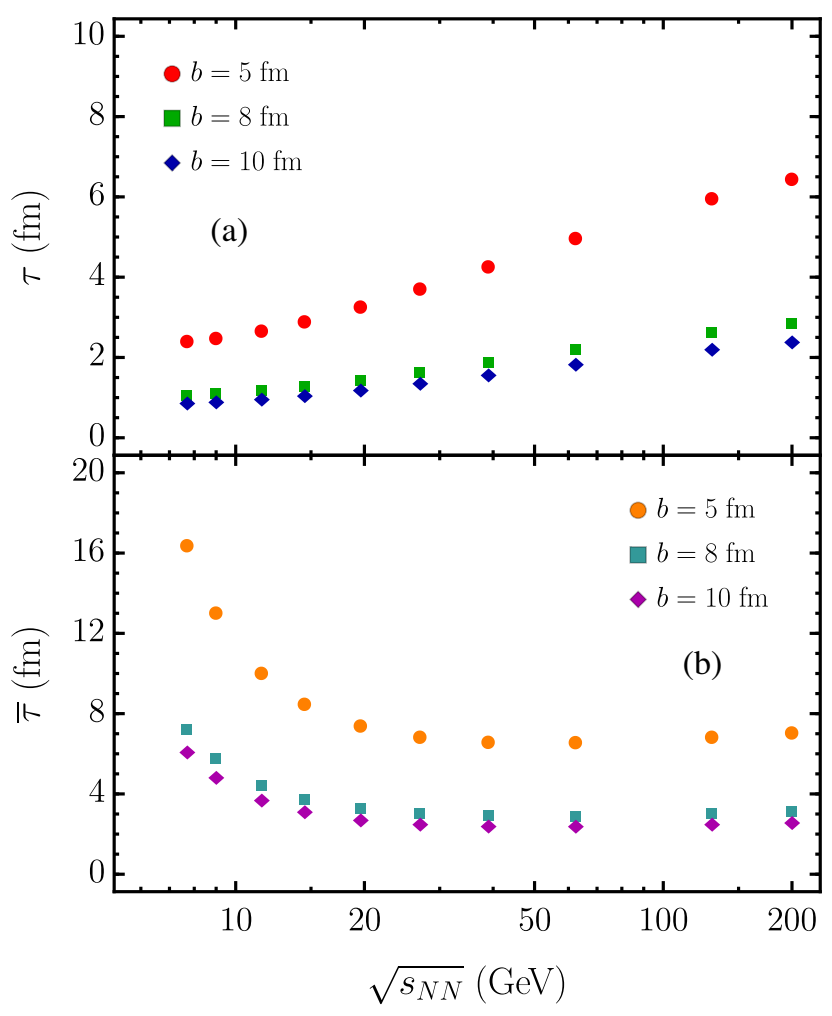

FIG. 6. (a) Relaxation time $\tau$ for quarks as a function of $\sqrt{s_{N N}}$ for semicentral collisions at impact parameters $b=5,8,10 \mathrm{fm}$. (b) Relaxation time $\bar{\tau}$ for antiquarks as a function of $\sqrt{s_{N N}}$ for semicentral collisions at impact parameters $b=5,8,10 \mathrm{fm}$.

relaxation times are smaller than the ones found in Ref. [20].

Figure 5 shows the temperature dependence of the relaxation time for antiquarks (with antiquark chemical potential $\bar{\mu}_{q}=-\mu_{q}$ ) for two different collision energies and chemical potentials at an impact parameter $b=10 \mathrm{fm}$. Again, we use for the antiquark mass $m_{q}=100 \mathrm{MeV}$, corresponding to the mass of the strange antiquark. The relaxation times for antiquarks for the temperature range $150 \mathrm{MeV}<T<200 \mathrm{MeV}$ satisfy $\bar{\tau} \lesssim 5 \mathrm{fm}$ and are also smaller than the corresponding relaxation times found in Ref. [20]. Figure 6 shows the relaxation time (a) $\tau$ for quarks and (b) $\bar{\tau}$ for antiquarks as functions of $\sqrt{s_{N N}}$ for semicentral collisions at impact parameters $b=5,8,10 \mathrm{fm}$ as a function of the collision energy. For each value of $\sqrt{s_{N N}}$, the temperature $T$ and maximum baryon chemical potential $\mu_{B}=3 \mu_{q}$ at freeze-out were extracted from the parametrization of Ref. [34] such that

$$
\begin{aligned}
T\left(\mu_{B}\right) & =166-139 \mu_{B}^{2}-53 \mu_{B}^{4}, \\
\mu_{B}\left(\sqrt{s_{N N}}\right) & =\frac{1308}{1000+0.273 \sqrt{s_{N N}}},
\end{aligned}
$$

where $\mu_{B}$ and $T$ are given in $\mathrm{MeV}$. Notice that the relaxation times for quarks show a monotonic growth as a function of 


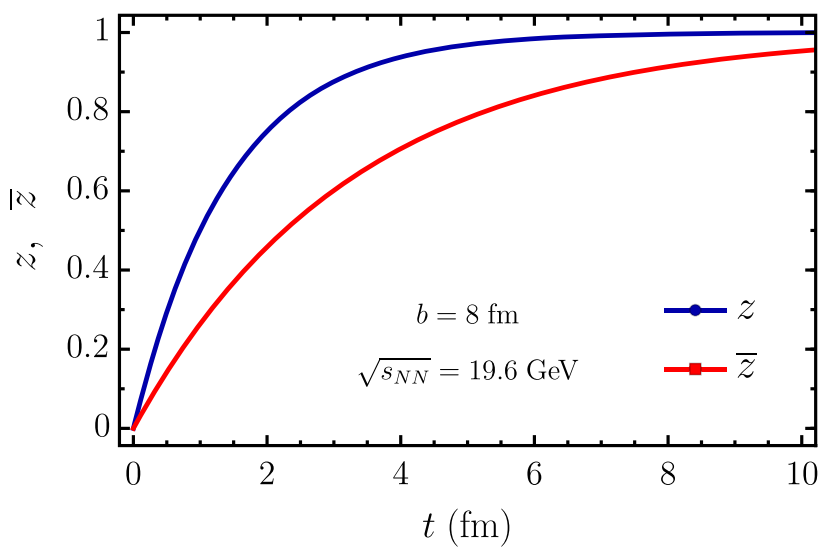

FIG. 7. Intrinsic global polarization for quarks $(z)$ and antiquarks $(\bar{z})$ as functions of time $t$ for semicentral collisions at an impact parameter $b=8 \mathrm{fm}$ for $\sqrt{s_{N N}}=19.6 \mathrm{GeV}$. Notice that $\bar{z}<z$; however, both intrinsic polarizations tend to 1 for $t \simeq 10 \mathrm{fm}$.

the collision energy throughout the energy range considered. In contrast, the corresponding relaxation times for antiquarks have a minimum for collision energies in the range $40 \mathrm{GeV} \lesssim \sqrt{s_{N N}} \lesssim 70 \mathrm{GeV}$ and grows slowly thereafter.

In order to use the relaxation times thus found to estimate the intrinsic global polarization induced by the alignment of the quark spin to the thermal vorticity, recall that when a number of particles $N_{0}$ initially occupying a given state (unpolarized spins) decays to occupy another state (polarized spins), the number of particles $N$ in the original state varies as a function of time $t$ as $N=N_{0} \exp (-t / \tau)$. Therefore, the number of particles coming out from this state (which corresponds to the number of particles that align their spins with the vorticity) is given by $N=$ $N_{0}[1 \exp (-t / \tau)]$. The factor $[1 \exp (-t / \tau)]$ can thus be properly called the intrinsic global polarization.

Figure 7 shows the intrinsic global polarization for quarks $(z)$ and antiquarks $(\bar{z})$, given by

$$
\begin{aligned}
& z=1-e^{-t / \tau}, \\
& \bar{z}=1-e^{-t / \bar{\tau}},
\end{aligned}
$$

as functions of time $t$ for semicentral collisions at an impact parameter $b=8 \mathrm{fm}$, for $\sqrt{s_{N N}}=19.6 \mathrm{GeV}$. Notice that $\bar{z}<z$; however, both intrinsic polarizations tend to 1 for $t \simeq 10 \mathrm{fm}$. From this figure, we also notice that, even if the QGP phase lasts for less than $10 \mathrm{fm}$, a finite intrinsic global polarization, both for quarks and antiquarks, can still be expected.

\section{SUMMARY AND CONCLUSIONS}

In conclusion, we have used a thermal field theoretical framework to compute the relaxation times for massive quarks and antiquarks (with a mass corresponding to the $s$-quark) whose spin interacts with the thermal vorticity produced in peripheral heavy-ion collisions. With the relaxation times at hand, we also computed the intrinsic quark and antiquark global polarizations. When this last is preserved during the hadronization process, one might expect that these polarizations directly translate into the corresponding $\Lambda$ and $\bar{\Lambda}$ polarizations. This would in turn imply that the former should be expected to be larger than the latter, as opposed to the findings of Ref. [18]. Contrary to these expectations, Ref. [35] has recently found that these intrinsic polarizations can give rise to the $\Lambda$ and $\bar{\Lambda}$ polarization experimentally observed as a function of collision energy. This result comes from the interplay of $\Lambda$ and $\bar{\Lambda}$ abundances when their source in the reaction zone is modeled as a composed of a high-density core and a less dense corona. Although both regions partake of the vortical motion, $\Lambda$ s and $\bar{\Lambda}$ s coming from one or the other could show different polarization properties as their origins are different: in the core they come mainly from QGP induced processes, whereas in the corona they come from $n+n$ processes. When this fact is combined with a larger abundance of $\Lambda \mathrm{s}$ as compared to $\bar{\Lambda} \mathrm{s}$ in the corona region together with a smaller number of $\Lambda$ s coming from the core as compared to those coming from the corona-which happens for semicentral to peripheral collisions-an amplification effect for the $\bar{\Lambda}$ polarization can occur. This is more prominent for small collision energies. More detailed studies of the effect are provided in Ref. [35].

The findings of this work provide a practical estimate for the relaxation times. The calculation makes use of parameters that can be obtained from thermal models and transport simulations. Notice that the angular velocity and the collision volume are quantities that vary with time. While a large volume, as it is expected at freeze-out, would reduce the relaxation time, an also expected smaller angular velocity at freeze-out produces an increment in the relaxation time. For the sake of simplicity and transparency of the results, we have assumed that the estimates for $\omega$ and $V$ yield an overall acceptable estimation, provided the quantity $V \omega^{2}$ varies little over time. More detailed studies that include the time evolution of these parameters are being performed and will be reported elsewhere.

\section{ACKNOWLEDGMENTS}

The authors acknowledge useful conversations with S. Hernández and X.-G. Huang during the genesis of this work. Support has been received by UNAM-DGAPAPAPIIT Grant No. IG100219 and by Consejo Nacional de Ciencia y Tecnología Grants No. A1-S-7655 and No. A1-S-16215. L. A. H. acknowledges support from a PAPIIT-DGAPA-UNAM fellowship. 
[1] F. Becattini, I. Karpenko, M. A. Lisa, I. Upsal, and S. A. Voloshin, Phys. Rev. C 95, 054902 (2017).

[2] Y. Xie, R. C. Glastad, and L. P. Csernai, Phys. Rev. C 92, 064901 (2015).

[3] A. Sorin and O. Teryaev, Nucl. Part. B, Phys. Proc. 273-275, 2587 (2016).

[4] A. Sorin and O. Teryaev, Phys. Rev. C 95, 011902(R) (2017).

[5] Y. L. Xie, M. Bleicher, H. Stöcker, D. J. Wang, and L. P. Csernai, Phys. Rev. C 94, 054907 (2016).

[6] H. Li, L.-G. Pang, Q. Wang, and X.-L. Xia, Phys. Rev. C 96, 054908 (2017).

[7] Y. Sun and C. M. Ko, Phys. Rev. C 96, 024906 (2017).

[8] Z.-Z. Han and J. Xu, Phys. Lett. B 786, 255 (2018).

[9] X.-L. Xia, H. Li, Z. Tang, and Q. Wang, Phys. Rev. C 98, 024905 (2018).

[10] M. Baznat, K. Gudima, A. Sorin, and O. Teryaev, Phys. Rev. C 97, 041902(R) (2018).

[11] I. Karpenko and F. Becattini, Nucl. Phys. A982, 519 (2019).

[12] D. Suvarieva, K. Gudima, and A. Zinchenko, Phys. Part. Nucl. Lett. 15, 182 (2018).

[13] E. E. Kolomeitsev, V. D. Toneev, and V. Voronyuk, Phys. Rev. C 97, 064902 (2018).

[14] Y. Xie, D. Wang, and L. P. Csernai, Eur. Phys. J. C 80, 39 (2020).

[15] Y. Guo, S. Shi, S. Feng, and J. Liao, Phys. Lett. B 798, 134929 (2019).

[16] H.-B. Li and X.-X. Ma, Phys. Rev. D 100, 076007 (2019).

[17] B. I. Abelev et al. (STAR Collaboration), Phys. Rev. C 76, 024915 (2007); 95, 039906(E) (2017).

[18] L. Adamczyk et al. (STAR Collaboration), Nature (London) 548, 62 (2017).
[19] J. Adam et al. (STAR Collaboration), Phys. Rev. C 98, 014910 (2018).

[20] A. Ayala, D. de la Cruz, S. Hernández-Ortíz, L. A. Hernández, and J. Salinas, Phys. Lett. B 801, 135169 (2020).

[21] J. I. Kapusta, E. Rrapaj, and S. Rudaz, Phys. Rev. C 101, 024907 (2020).

[22] S. Y. F. Liu, Y. Sun, and C. M. Ko, arXiv:2002.11752.

[23] S. Shi, C. Gale, and S. Jeon, arXiv:2002.01911.

[24] Z.-T. Liang and X.-N. Wang, Phys. Rev. Lett. 94, 102301 (2005); 96, 039901(E) (2006).

[25] A. Aristova, D. Frenklakh, A. Gorsky, and D. Kharzeev, J. High Energy Phys. 10 (2016) 029.

[26] L.-G. Pang, H. Petersen, Q. Wang, and X.-N. Wang, Phys. Rev. Lett. 117, 192301 (2016).

[27] J.-H. Gao and Q. Wang, Phys. Lett. B 749, 542 (2015).

[28] F. Becattini, F. Piccinini, and J. Rizzo, Phys. Rev. C 77, 024906 (2008).

[29] F. Becattini, G. Inghirami, V. Rolando, A. Beraudo, L. Del Zanna, A. De Pace, M. Nardi, G. Pagliara, and V. Chandra, Eur. Phys. J. C 75, 406 (2015).

[30] X.-G. Deng, X.-G. Huang, Y.-G. Ma, and S. Zhang, Phys. Rev. C 101, 064908 (2020).

[31] S. A. Bass et al., Prog. Part. Nucl. Phys. 41, 255 (1998); M. Bleicher et al., J. Phys. G 25, 1859 (1999).

[32] Y. Jiang, Z.-W. Lin, and J. Liao, Phys. Rev. C 94, 044910 (2016); 95, 049904(E) (2017).

[33] W.-T. Deng and X.-G. Huang, Phys. Rev. C 93, 064907 (2016).

[34] J. Cleymans, H. Oeschler, K. Redlich, and S. Wheaton, Phys. Rev. C 73, 034905 (2006).

[35] A. Ayala, M. A. Ayala Torres, E. Cuautle, I. Domínguez, M. A. Fontaine Sánchez et al., arXiv:2003.13757. 